XV.

\title{
Die Ohrenentzündungen in ihren abhängigen Beziehungen zu Nachbarorganen,
}

\author{
Fine Skizze \\ ron \\ L. Grînwald (Bad Reichenhall-München).
}

\section{Verehrter Altmeister!}

Nicht weit noch liegt die Zeit zurück, da eine Ohreneiterung nicht viel mehr denn einen unangenehmen "FluB" bedeutete.

Ihr Verdienst ist es, der rein chirurgischen Auffassung der Obrenentzündungen Bahn gebrochen zu haben, als deren letzte Stufe unsere heutige Erkenntnis dasteht, daß bei endocraniellen septischen Prozessen in erster Linie die Beschaffenheit des Ohres kontrolliert werden muß.

Der so geschaffene Vorstellungskreis teilt aber mit allen menschlichen Assoziationsgebieten die Gefahr, zu weit zu reichen, zuviel zu decken.

Die Gefahr ist, ich werde das in den folgenden eigenen und fremden Erfahrungen nachweisen, nicht imaginär; sie ist mitunter sogar groß, weil der irrtümlich als vorwiegend oder allein vorhanden angesehene Ohrprozeß andere, sogar letale Grunderkrankungen zu verdecken imstande ist.

Auch in dieser Richtung den Ohrenerkrankungen ihren chirurgisch richtigen Platz anzuweisen, ist sicher in Ihrem Sinne, verehrter Altmeister, gehandelt.

\section{A. Durchbruch von Eiter aus Nachbarorganen.}

Es ist bekannt, daß otitische extradurale und Hirnabszesse durch das $\mathrm{Obr}$ ibren Abflub finden können. Diese Ereignisse interessieren uns hier weniger: meist wird es früher oder später 
gelingen, das Bestehen des endocephalen Herdes nachznweisen, an dessen Ausheilung außerdem die Behandlung der Obreneiterung einen integrierenden Bestandteil darstellt. Anders jene, allerdings überaus seltenen Fälle von

\section{Abflub nicht otitischer endocephaler Abszesse durch das 0 hr.}

1. Le Blanc (1). Nach einem Schlag auf den Kopf entstand nach vorubergehender Bewubtlosigkeit ein sich stetig steigernder intensiver Kopfschmerz, zu welchem sich nach ca. 8 Tagen klonische und tonische (allgemeine?) Krämpfe und Schlaflosigkeit gesellten. Nach vorïbergehender Besserung, vom 15.-30. Tage, lokalisierte sich wiederum ein enormes Druckgefühl in der Gegend zwischen Pfeilnaht nnd linkem Ohr, bis in der Nacht zum 56. Tage nach vorgängigem Schmerznachlab der änherst heruntergekommene Kranke einschlief und beim Erwachen, nach einer halben Stunde, das Kopfkissen von noch in einem anbaltenden feinen Strahl aus dem linken ohr sich entleerenden Eiter durchtränkt fand; der Ausfluß hielt dann, vermindert, noch einige Wochen an, während deren none Schmerzen auf der rechten Seite auftraten, bis anch aus $d e m$ rechten $\mathrm{Ohr}$ am 86. Tage Eiter austrat. Intermittierend auftretende Gesichtszuckungen versehwanden jedesmal nach Abflab einiger Eitertropfen aus dem linken Ohr. - Tach einer kleinen Verschlechterung von kürzerer Dauer (Entstehung einer Geschwulst hinter dem linken Ohr, welche nach reichlicherem Austlu $B$ wieder versehwand, kehrten nach ca. 31/4 Jahren plötzlich die Kopfschmerzen, begleitet von Übelbefinden, Schnupfen, Anosmie und Appetitlosigkeit ete. wieder, bis eines Nachts eine groBe Menge über die Maßen stinkenden Eiters ans dem Nasenrachenranm per os entleert wurde, woranf rasch danernde Genesung eintrat.

Eine nähere Charakterisierung des Prozesses, ob cerebral, subdural oder extradural, ist kaum möglich, sicher wird er nur, durch Erscheinungen und Verlauf, als end ocephal gekennzeichnet; ein Fortkriechen unter der Scbädelbasis würde andere Symptome hervorrufen.

2. Rust (2): Nach einem Sturz (vom Pferd) auf den Kopf stellte sich sich ein periodisches halbseitiges Kopfweh von soleher Intensität ein, daß dje geistige Beschäftigung (welche die Anfälle hervorzurufen schien), aufgegeben werden multe. Nach einem neuerlichen Sturz (13 Jahre später), zeigte sich ans dem linken Ohre ein Abflup von Blut und klarem Eiter", der einige Tage anhieit, mehrere Unzen (à $30 \mathrm{ccm}$ ) betrug und nach dessen Aufhören dauernde volle Genesung eintrat.

Wahrscheinlich traumatischer Extraduralabszeß, durch eine Basisfissur beim zweiten Sturz eröffnet.

3. Gama (3): Hufschlag auf den hinteren Rand des rechten Scheitelbeins nebst Sturz auf den Kopf. Nach vorïbergehendem BewuBtseinsverlust am 9. Tage bei der Aufnahme: Somnolenz, Blässe des Gesichtes und Lähmung des linken Gesichtes und Armes: mühsame Atmung, Puls beschleunigt; am nächsten Tage tiefes Coma. wieder.

Erst am 16. Tage kehrte das Bewußtsein, sowie die volle Motilität

Am 29. Tage schmerzhafte "Otitis" rechts, vom 32.-34. Tage überaus reichlicher Ausflub von wässrigem, sehr stinkendem Eiter aus dem Ohr, der dann rasch wachließ und mit voller Genesung (Entleerung am 45. Tage) endete. 
Der Verlauf in den ersten zwei Wochen ist nur unter der Annahme eines Hirndruckes durch ein langsam entstandenes Extravasat zu erklären. Ob dieses durch eine interkurrente Otitis auf dem Wege einer minimalen Fissur infiziert wurde oder, primär auf ebensolche Weise vereitert, beim Durchbruch in das Ohr erst die Erscheinung einer Otitis hervorgerufen hat, ist nicht entscheidbar, aber anch gleichgültig: an der endocephalen Bildung der Flüssigkeitsansammlung läßt sich kaum zweifeln.

Ein weiterer Fall ähnlicher Art ist derjenige von

4) M. 'Leod, den Barr (4), allerdings sehr kurz, referiert:

Ein traumatisch entstandener Vorderlappenabszeß kroch an der Schädelbasis entlang und brach im äußeren Gehörgang durch.

Endlich die sehr instruktive Beobachtung von

๖. Panas (5): Ein 21jähriger Mann erkrankte im Gefolge eines Erysipels an rechtsseitiger Orbitalphlegmone. Ca. 8 Wochen nach Beginn des Erysipels entstanden Schmerzen im rechten $\mathrm{Ohr}$, Perforation und anhalteade Eiterung. Nachdem bald darauf einige epileptische Anfälle aufgetreten waren, stellte sich eine allmählich bis zur Fluktuation gedeihende Schwellung über der Schuppen-Schläfengegend ein, die nach der Inzision zwei Perforationen des Knochens enthüllte. Nach dem, im 6. Monate der Erkrankung, im Coma eingetretenen Tode fand sich bei der Sektiou der ganze „Keilbeinlappen“ (,corne sphénoidal") in einen Eiterbrei verwandelt, die Meningen in der ganzen fossa sphenoïdalis adhärent, die Dura intakt, aber außer den 2 Löchern im Schuppenteil noch ein weiteres im tegmen tympani. Meningitis der Vorderlappenbasis bis zum Bulbus nach vorne reichend.

Hier liegt also wiederum Durchbruch einer Hirneiterung vor, im Anschluß aber ist eine echte sekundäre Otitis entstanden.

\section{Beteiligung des Obres an suboceipitalen}

\section{Abszessen.}

1. Eigener Fall. Aus der a. a. 0. (6) ansführlich wiedergegebenen Krankengeschichte hebe ich nur folgendes, hier interessierende hervor:

Der dureh Vereiterung einer abnorm gelegenen Keilbeinhöhle entstandene intravertebrale Absze $B$ an den beiden ersten Halswirbeln trat zunächst in Gestalt einer akuten Ohren-Warzenfortsatzeiterung zutage, welche allerdings in ibren Erscheinungen: profusester Absonderung trotz allen Freilegungsversuchen, Schwellung in der Gegend des emissarium Santorini, Koptsperre, Trigeminus und Cervicalneuralgie, sowie besonders in der Stelle des Eiterabflusses (am Boden) usw. verdächtige Abweichungen vom Typus darbot, sodass zunächst an Fortkriechen eines otitisch entstandenen perisinuösen Abszesses durch das emissarium gedacht wurde (s. auch u. 11, 12) Der Durchbruch des suboccipitalen Abszesses erfolgte hier im recessus infratympanicus. Zur näheren Instruktion muß ich auf die Originalmitteilung verweisen.

2. Das „malum suboccipitale", auf tuberkulösen Entzündungen der Atlanto-occipitalgelenke beruhend, löst, besonders im Anfang, mitunter Erscheinungen von Otitis aus, die einer Verschleierung des wahren Prozesses dienlich sind: 
E. v. Bergmann (7) berichtet über zwei solcher Fälle, in deren erstem die durch Lucae vorgenommene Parazentese die im Vordergrunde des Bildes stehenden Obrenschmerzen verminderte, während der Abszeß semen Ausweg retropharyngeal suchte; im zweiten entstand sogar eine vorübergehende Eiterung des Ohres und bald darauf eine faustgroße retroaurikulare Schwellung, die jedoch in ihren kausalen Beziehungen richtig erkannt wurde. Diese beiden letzteren Fälle sind natürlich nur als Korrelate zu den eigentlichen Durchbruchsprozessen und mehr im Sinne der weiter unten zu besprechenden konsensuellen Entzündungen aufzufassen.

III. Durehbruch von Parotis- und Kieferabszessen.

Für diese Eventualität stehen mir keine Einzelbeispiele zur Verfügung. Nur soviel:

Parotitische Phlegmonen bedrohen den äußeren Gehörgang und können durch die daselbst zunächst auftretende Anschwellung und das Macerationsekzem täuschende Bilder erzengen.

Über Kiefergelenkentzündungen äußert sich $\mathrm{Hy}$ rtl (8) dahin, daß der Eiter durch die fissura Glaseri oder die nicht selten offene Dehiszenz in der vorderen Wand des änBeren Gehörganges in die Trommelhöhle gelange.

Dentale Parulitiden vermögen denselben Weg, eventuell auch durch die fossa pterygo-palatina hindurch, einzuschlagen. -

Für alle Fälle der Gruppe A gilt folgendes:

Erstens mulb das Bestehen der als eigentlicher Eiterherd anzusprechenden Erkrankung durch dentliche, für sich sprechende Symptome resp. Befunde erwies en sein. Diese hier zu erörtern, verbietet der Raum. Bezüglich der suboccipitalen Entzündungen verweise ich speziell auf meine letzte Publikation (9).

Zweitens muß der zeitliche Verlanf sowohl als die Möglichkeit kausaler Verknïpfung einerseits der Ohrenerkrankung, andererseits dem Nachbarherd den richtigen Platz anzuweisen gestatten. In dieser Beziehung ist daran zu erinnern, daß so wie Hirnabszesse gewöhnlicher durch Ohreneiterungen hervorgerufen zu werden pflegen, als umgekehrt, letztere, allerdings seltener, ebensogut ihrerseits in suboccipitalen Entzündungen resp. periartikulären Eiterungen zu enden vermögen. Am klärendsten wird da zunächst die genaue zeitlich e Kontrolle der Einzelerscheinungen einzusetzen haben. 
Diese Kontrolle wird besonders dann Klarheit zu schaffen vermögen, wenn einer der seltenen Fälle otogener Entstehung: eines suboccipitalen Abszesses, speziell auf phlebitischem Wege, in Frage steht.

Auch ist an dieser Stelle daran zu erinnern, dah sogar der Umweg über das Hirn resp. den Extraduralraum seitens otitischer und andersartig entstandener Herde beschritten werden kann, um vermittels Durchbruches oder durch vorgebildete Wege (foramen condyloideum, emissarium etc.) im Nacken zu erscheinen $(10,11)$.

Endlich sollten, bei zunächst isolierten oder doch im Vordergrunde des Bildes stehenden Ohreneiterungen, abnorme Reichlichkeit und Hartnäckigkeit der Sekretion, besonders aber jedes nicht ganz gewöhnliche Symptom auf die Möglichkeit anderer Substrate aufmerksam machen. Hier nenne ich speziell abnorm lokalisierte Schmerzen, auffallende Lokalisationen von Schwellungen, ebensolche Bewegungsbehinderungen (Kiefer- oder Kopfsperre), endlich gewisse motorische Läbmungserscheinungen, speziell im Bereiche des n. abducens, occulomotorius, trigeminus, accessorius und hypoglossus.

\section{B. Consensuelle und sekundäre Ohreiterungen.}

Der Einfluß benachbarter Erkrankungen braucht sich nicht bis zum Durchbruch der Eiterung zu erstrecken; Infektion, auf verschiedenen Wegen vermittelt, kann neben der ursprünglichen Erkranknng einen Reizzustand, ja mehr als das, eine bis zur Eiterung gehende Entzündung im Ohr vermitteln, deren Abhängigkeit von jenen, also rein sekundäre Bedeutung, im Verlaufe erkannt werden kann und, genau wie bei Durchbrïchen, dahin zu würdigen ist, daß die Oh renerkrank ung ohne Beseitigung. des primären Herdes keine Heilungsausichten bietet, daß sie ferner diesen Herd zu verdecken and dadurch die Heilungsmaßnahmen von der richtigen Bahn abzulenken imstande ist, endlich daß die konsensuelle Form nach Auschaltung des primären Herdes ohne weiteres von selbst versehwindet.

Für all diese Eventualitäten bieten die folgenden Fälle Belege:

1. Finlag (12): Bei einem 16jährigen Jüngling bestand seit mehreren Wochen linksseitige Ohreneiterung, seit zwei Tagen heftige Schmerzen daselbst, Die Eiterning schwankte stark in der Menge, war zeitweise profus. Status: Halbcoma mit D elirien, T. 39,9, P. 120 . Links kJeine Perforation, wenig Eiter, links A bducenslähmung; Augenhintergrund normal. 
Während der Narkose bemerkte man eine parallel dem linken oberen Orbitalrand, etwas nnterhalb, verlaufende blutegelförmige Anschwellung und stellte die richtige Diagnose auf Phlebitis des sinus cavernosus. Trotzdem wurde nur auf das Ohr vorgegangen: Im Antrum und Mittelohr fanden sich nur winzige Mengen von Eiter; der Knochen, ebenso der sinus transversus, die Dura und das Hirn erwiesen sich als intakt. Am nächsten Morgen Exitus.

Bei der Sektion fanden sich die Ohräume eiterfrei, die Dura an der Basis dickplastisch infiltriert, in den sinus cavernosus und circularis ein ,eitriges Blutcoagulum", bis in die linke v. ophthalm. (sup.) reichend, Keil bein und Siebbeinzellen mit dickem gelbem Eiter gefült.

F. erkennt nachträglich selbst die rein sekundäre Bedeutung: der Ohrenaffektion an, hält es jedoch, in Anbetracht der tatsächlich bestandenen Otitis, trotz den auf den sinus cavernosus hindeutenden Symptomen für wmmöglich, anders zu handeln.

Ob diese Annahme, allein schon gegenüber der von uns prinzipiell erhobenen Forderung, jeden Obrenfall anch in der Nase zu untersuchen, aufrecht erhalten werden könnte?

2. Uchermann (13): Einem 18jährigen Mädchen war ror 4 Jahren ein Abszeß des Zahnfleisches in den rechten Gehörgang durchgebrochen, dann waren Drüsenabszesse, erst links und dann unter dem rechten $0 \mathrm{hr}$ gefolgt, jedoch alles geheilt.

Wenige Tage vor der Aufnahme entstand unter Schüttelfrost eine schmerzhafte Anschwellung hinter dem linken Kopfnickeransatz; Trommelfell nur leicht gerötet, Warzenfortsatz etwas druckempfindlich, kein Ohrenfluß.

Die Operation enthüllte einen nach der Schädelbasis zu gelegenen AbszeB hinter und unter dem proc. mast. Die Schleimhant in letzterem hyperämisch, an der Spitze eiuige vereiterte Zellen. Sinus transv. frei.

Neue Schüttelfröste und Temperaturanstieg bis zu $41,6^{\circ}$ veranlaßten zu weiterer Freilegung des sin. transv. und der v. jugul., ohne Ergebnis. Tod nach 2 Tagen.

Bei der Sektion fand sich der sinus occipitalis, bis zum confluens hin, samt den Cervical(Occipital?) venen thrombosiert. Hirnödem.

U. sieht in dieser Erkrankung die Folge einer Infektion seitens der periphersten Zellen.

Wenn man aber seinen Gesichtskreis nicht nur vom otologischen Standpunkt aus übersieht, so stellt sich der wahrscheinliche Verlauf (ohne weitere Ausdehnung der Sektion läbt sich keine größere Gewißheit erlangen) folgendermaßen dar:

Eine dem ursprünglichen Herd nahegelegene oder mit ihm identische Infektionsquelle in der Nachbarschaft der fossa pterygo-palatina (Zähne, Nebenhöhlen usw.) hat über den plexus pterygoideus zu einer abszedierenden Phlebitis der Occipitalvenen geführt; die Verbindungen der v. facial. post. mit den Paukenwarzenfortsatzvenen vermittelten eine rein syndromatische Beteiligung der Warzenfortsatzspitze, während andererseits die Infektion ihren Hauptweg weiter zum sinus occipitalis nahm.

Die Ohrenoperation konnte an sich weder diesen Verlauf enthüllen noch unterbrechen; ihr negatives Ergebnis hätte aber 
auf die Möglichkeit anderweitiger Zusammenhänge hinweisen müssen, wenn nicht schon die Anamnese und der eigentümliche Befund auf eine solche aufmerksam gemacht hätten.

Klinischen Schwierigkeiten durch raschen tödlichen Verlauf enthoben, bietet der Fall von

3. Konietzko-Isemer (14), dessen ausführlichem interessanten Bericht ich nur die hier wichtigsten Schlagworte entnehme, unsomehr des fraglos Belehrenden:

Alte Eiterung der linken Kieferböhle mit Ausgang in Nekrose. Perforation einerseits zum harten Gaumen, andererseits in der hinteren oberen Wand zur fossa pterygo-palatina. Gangrän der Weichteile daselbst sowie des perivaskulären Gewebes in canalis carotid., des peritubären Gewebes und des m. tensor tymp., endlich der Schleinhaut um den Steigbügel.

Arrosion des Hinterhauptbeins bis über den linken prozessus condyloid. hinans uud bis über die Mittellinie nach rechts.

Die Wände der vena und des bulbus jugul. bereits verfärbt, die Gefäße innen noch intakt; einzelne Drüsenpakete am Halse verfärbt, ihre Ungebung sulzig infiltriert.

Eiter in der Paukenhöhle, die Gehörknöchelchen von Granulationen umgeben, auf deren Frische gegenüber den nekrotischen, primär erkrankten Partien die Autoren ausdrücklich hinweisen, um daran selbst die rein sekundäre Bedeutung der Otitis zu erläutern.

4. Eigen er F all (15). Aus der Krankengeschichte, deren ausführliche Lektüre zurWürdigung der diagnostisch entscheidenden Momente des Falles unerläßlich ist, hebe ich nur folgende Daten kurz hervor:

Exacerbation eines alten Kieferhöhlenkatarrhs. Breite Eröffnung vom unteren Nasengange aus. 14 Tage später neuerliche Infektion (Erkältung). Fortschreiten der Entzündung durch die Hinterwand der Kieferhöhle auf das Gewebe der fossa pterygopalatina nnd anf das suboccipitale Gewebe, gleichzeitig, wahrscheinlich auf dem Lymphwege, zum Ohr. Profuse Mittelohreiterung mit hoher Empfindlichkeit der Warzenfortsatzspitze. Diagnose eines beginnenden suboccipitalen Abscesses auf Anamnese, leichte Kiefersperre, tiefes Nackeninfiltrat. Kopfsperre und Neuralgie der 2. und 3. Trigeminusastes begrüindet.

Operation des Abseesses mit Freilegung der hinteren, ganz gesund aussehenden Warzenfortsatzilläche.

Nach Entfernung des Wundtampons am 4. Tage, Versiegen der bis dabin profus andauernden Ohreneiterung innerhalb w: niger Stunden.

In diesem Falle hätte in Anbetracht der fünf Wochen langen Dauer der profusen Ohreneiterung und der Schmerzhaftigkeit des processus nichts näher gelegen, als die pneumatischen Räume zu eröffnen, die sicher auch, zur Befriedigung des Operateurs, Eiterinhalt gezeigt haben würden. Ich habe, entgegen begründeten Einwänden sachkundiger Kollegen, fest auf der Auffassung einer nur sekundären Bedeutung der Obreneiterung bestanden und der postoperative Verlauf hat dem recht gegeben. Es ist dies der erste Fall, in dem es gelang, unter Abstraktion von den täuschen- 
den Ohrenerscheinungen sofort auf den richtigen Herd loszugehen.

In einem, viel früher, von mir ebenfalls als nicht otitisch begutachteten Falle ist von anderer Seite operiert worden. Darüber weiter unten.

Es existieren noch einige, meiner Ansicht nach hierhergehörige Fälle in der Literatur, speziell einer von $\mathbf{J}$ a $\mathrm{n}$ s e $\mathbf{n}(16)$, auf die ich aber nicht eingehen kann, weil die ausführliche, zu der erforderlichen kritischen Würdigung unerläBliche Wiedergabe hier zu viel Platz beanspruchen würde. In anderem Zusammenhange werde ich dieselben ohnedies noch zu besprechen haben.

Nur einen möchte ich, des besonderen Interesses halber, kurz erwähnen, denjenigen von

2. Neumann (17): Nach einer Nasenoperation setzte eine acute Otitis ein, die am 6. Tage duch Schüttelfrost und hohes Fieber kompliziert wurde, aber keine anderen subjektiven oder objektiven Zeichen von Mastoiditis darbot. Trotzdem wurde die Aufmeißelung vorgenommen, „die aber keine Aufklärung brachte", weiter noch die jugularis unterbunden und der Bulbus freigelegt, ebenfalls ohne positiven Befund; speziell kein obturierender Thrombus.

Nach der Operation hörten die Schüttelfröste auf und sank die Temperatur lytiseh ab.

$\mathrm{N}$. nimmt an, daß eine wandständige, bei der starken Blutung nicht sichtbare Thrombose des Querleiters vorgelegen habe.

Allerdings ist das Vorhandensein einer Otitis geeignet, dieser Auffassung eine Stütze zu leihen.

Trotzdem ist dieselbe abzuweisen. Der absolut negative Ausfall der Operationen verlangt das; die Entzündung beschränkte sich ja auch nur auf die Paukenhöhle.

Höchstwahrscheinlich sal der Thrombus in einem der sinus petrosi, vielleicht auch nur im plexus pterygoideus. Die Iugularisunterbindung unterbrach die Blutverschleppung weiteren septischen Materials, konnte aber nicht verhindern, daß von dem noch bestehenden, jedenfalls sehr kleinen Herde aus noch septische Stoffe resorbiert wurden, weshalb das Fieber nicht sofort, sondern nur lytisch abfiel.

Die Phlebitis war zweifellos direkt nasalen Ursprungs, die Otitis ein Syndrom.

Ihre Entstehung zu erörtern, ist im knappen Rahmen dieser Skizze nicht möglich; unzweifelhaft ist nur, daß auch hier, wie in mehreren der bereits erörterten Fälle, der Inhalt der fossa pterygo-palatina und insbesondere der plexus pterygoidens eine 
ausschlaggebende Vermittlerrolle spielt, deren prinzipi elle Bedeutung usw. zurzeit von mir ausführlichen Untersuchungen unterzogen wird.

Eine eigentümliche und nur durch Anwendung aller einschlägigen Untersuchungsmethoden, insbesondere wieder dureh systematische Nasenuntersuchung vermeidbare Fehlerquelle besteht in der

\section{Gleichzeitigkeit von 0hreneiterungen und andersartigen Schädeleiterungen.}

1. Ko eb el (18): Bei einem 39 jährigen Manne, der von Jugend auf mit Naseneiterung behaftet war, trat ein Recidiv einer 15 Jahre vorher entstandenen rechtsseitigen Ohreneiterung auf. In der rechten Nase wurden Granulationen geätzt; 14 Tage daruuf entstand plötzlich nnter Fieber ein heftiger Schmerz in der Stirn und beiden Schläfen neben allgemeinem Verfall, schwankendem Gang, (wie in Betrunkenheit), Erbrechen, Unruhe und, einige Tage später, Zuckungen in der linken Hand und Arm. Stirn nicht druckempfindlich.

Die Totalaufmeißelung ergab keine Knochenerkrankung, anch nicht der ossicula; Eiter im Antrum.

In anhaltendem Sopor trat bald dor Tod ein.

Bei der Sektion fand sich eine Perforation in der Hinterwand der rechten Stirnhöhle (beide, auch die linke, von Eiter und Granulationen erfüllt) und ein hühnereigroßes Abszess im Vorderlappen.

Daß die Ohreneiterung die Dignität des gleichzeitigen Nasenprozesses stark zu verhüllen geeignet war, ist klar. Die Druckunempfindlichkeit der Stirn konnte umsomehr zur Täuschung beitragen, als, entgegen meinen wohlbegründeten Warnungen (23), seit der Kuhntschen Arbeit, diesem Symptom ein viel zu weitgehendes Gewicht beigelegt wird; das einzige, auf den Vorderlappen hinweisende Symptom der "frontalen Ataxie" ist nicht auffällig genug, um seine Vernachlässigung nicht begreiflich zu finden.

Trotzdem oder vielmehr gerade darum bietet der Fall erneuten Anlaß, unsere Mahnung zu noch größerer Aufmerksamkeit auf die Nase usw. zu wiederbolen. Gerade solche Fälle mit unklaren, aber doch auf endocephale Vorgänge hinweisenden Symptomen, müssen zur Erschöpfung aller Möglichkeiten durch Anwendung weitgehender Untersuchungen führen und speziell zur Anwendung der Erkenntnis, daß latente Nebenhöhleneiterungen häufig nur den intensirsten diagnostischen Bemühungen zugängig sind.

2. In Stenger's (19) Fall beherrschte die Stimhöbleneiterung, da sie bald in Anfange zu einem Lidabszess führte, von vornherein die cbenfalls durch eine subakute Ohreneiterung komplizierte Lage. Demgemäß wurde auf der richtigen Linie vorgegangen, allerdings nicht weit genug: der unentdeckte Stirnlappenabszess führt zum Tode. 
Wenn in der Koebelschen Beobachtung die Gleichzeitigkeit der Ohrenaffektion mit der tödlichen Stirnhöhleneiterung die Täuschung über den wahren Sachverhalt in vivo noch verständlicher machte, umsomebr als auch die Lokalhirnsymptome geradeso gut von einem Schläfenlappenabszeß ausgelöst sein konnten, so hätte in dem folgenden Fall von

3. J a n s e n (20) die Möglichkeit einer anderen Aufassung, wenigstens post mortem, nahegelegen:

In der rechten Nase eines mit alter lin kseitiger desquamativer Ohreneiterung behafteten Mannes wurde galvanokaustisch gebrannt. Allgemein septische Erscheinungen traten aul und veranlaBten die linksseitige Aufmeißelung, führten aber 2 Tage später zum Tod.

Bei der Sektion fandeu sich sämtliche Blutleiter frei, nur in dem rechten sinus petros inf. ein eitrig zerfallender, grünlich verfärbter Pfropf, ebenso in der sella tureica miffarbige zerfallene Massen, am clivus der Knochen grünlich verfärbt. - Keine Nebenhöhlensektion.

Daß die linksseitige Ohreneiterung diese rechtsseitige Basiserkrankung, unvermittelt noch dazu, herbeigeführt haben sollte, ist absolut von der Hand zu weisen. Die Entstehung, im Anschluß an die Galvanokaustik, weist in ganz typischer Weise wieder auf eine nasale Entstehung der Phlebitis hin.

Immerhin, auch in diesem letzten Falle lag doch wenigstens noch eine manifeste Ohreneiterung, wenn auch der anderen Seite, vor.

Es bleibt uns aber noch übrig, eine Gruppe von Fällen zu besprechen, in denen überhaupt keine Otitis bestand, sondern nur durch lokale oder allgemeine Symptome, welche bei Ohreneiterungen wohl vorkommen, aber auch anderen weniger bekannten Erkrankungen eigentümlich sind, vorgespiegelt wurde.

\section{Vortäuschung nicht vorhandener Ohreneiterungen.}

In dem ersten der einschlägigen Vorkommnisse schöpfte der Operateur wenigstens aus dem Umstande, dab eine Ohreneiterung relativ kurze Zeit rorhergegangen war, einige Berechtigung für seine Auffassung:

1. Eigener Fall (21): Geheilte Kopfverletzung vor 6 Jahren; vor einem halben Jahre linksseitige, rasch geheilte Ohreneiterung.

Heftige Schmerzen hinter dem linken Ohr und Beschränkung der Kopfbeweglichkeit führten den behandelnden Arzt dazu, mir den Patienten als der Simulation verdächtig (Unfallrente) vorzustellen.

Ich konnte absolut keine objektiven Veränderungen, auch nicht am Ohre, feststellen und lehnte jedes Einschreiten, aber auch ein definitives Urteil ab.

Das Anhalten der Beschwerden und eine occipitale Schwellung veranlaßte den Kollegen, den Warzenfortsatz aufzumeileln, der sich als nicht erkrankt erwies, aber auf dem plaum eine alte Fissur mit eingeklemmter Fascie zeigte.

Arohiv f. Ohrenheilkunde. 73. Bd. Festsehrift. 
Einen Monat später wieder zugezogen, war ich in der Lage, den Proceß als einen rein suboccipitalen (es fand später noch eine retropharyngeale Senkung etc. statt) anzusprechen und zu operieren. Die akute otitis war selbst geheilt, hatte aber durch die, jedenfalls auch auf die Innenseite des processus reichende Fissur, die Infektion suboccipital geleitet.

Bezüglich der Einzelheiten verweise ich auf die Originalmitteilung.

Die Täuschungsmöglichkeit in diesem Falle war groß und wohlbegrïndet.

Im Gegensatz hierzu müssen wir auf die Notwendigkeit, sehon im klinischen Verlauf andere Möglichkeiten in Erwägung: zu ziehen, für folgende Fälle hinweisen:

2. Biehl (22): Im Gefolge einer Nasenoperation entstanden Ohrenschmerzen, die zu fünfmaliger, immer vergeblicher Paracentese Anlab gaben. Da abendliche Temperatursteigungen, einmal sogar Schüttelfrost auftraten, wurde weiter vorgegangen, immer in der Richtung anf das Ohr: der Warzenfortsatz aufgemeißelt und leer befunden; endlich fand sich ein kirschkerngroßer Abszess am Ubergang des sinus transversus zum sinus sigmoideus.

In einem zweiten Fall führten ebenfalls nach einem Naseneingriff aufgetretene Ohrenschmerzen zur ergebnislosen Ohrenoperation, die aber wiederum die Freilegung eines Sinusabszesses ermöglichte.

Auch epikritisch beharrt B. auf der Auffassung otitisehen Ursprungs dieser Abszesse, obgleich es doch sehr schwer ersichtlich ist, wie eine erweislich nicht existierende Ohrenentzündung Folgeerscheinungen hervorrufen soll.

Berechtigt seheint mir wiederum nur eine Erklärung, daß ähnlich wie in den oben erörterten Fällen dureh den Naseneingriff eine infektiöse Phlebitis der vena nasalis posterior veranlaßt und vermittels des plexus pterygoideus zu einem sin. petrosus und durch diesen zum sinus sigmoideus geleitet wurde.

(Bei dieser Gelegenheit möchte es nicht unwichtig sein, darauf hinzuweisen, daß Sinusthrombosen im Gefolge akuter Erkrankung an sich sehon den Verdacht auf einen anderen als otitischen Ursprung hinzulenken geeignet sind; nach akuten Ohrenerkrankungen kommen sie weitaus seltener zur Beobachtung; sehr erklärlich durch die fast unerläßliche Notwendigkeit vermittelnder Knochenprozesse, wenn es sich um das Obr handelt.)

Das einzige wirklich oder vielmehr scheinbar auf die Ohren hindeutende Symptom, der Ohrenschmerz ist ferner genau so trügeriseh, wie jede andere spontane Sehmerzlokalisation.

Auf die Hinfälligkeit aller aus diesen Lokalisationen gezogenen Schlußfolgerungen habe ich schon vielfach hingewiesen (23) und stehe damit ja nicht allein da (24). 
Was speziell den Ohrenschmerz und dessen unberechtigte Würdigung anbelangt, verweise ich auch auf eine jüngst erfolgte Zusammenstellung von komplizierenden Erkrankungen bei Keilbeinhöhleneiterung, durch St. Clair Thomson (25), aus welcher ersichtlich ist, dab Schmerzen hinter dem Ohr in einem, im Ohr selbst in 5 Fällen letzterer Art geklagt wurden und zwar so ausgesprochen, daß dreimal (vergeblich) der Warzenfortsatz eröffnet wurde und zwar von "geschickten Otologen." Sogar der sinus sigm. wurde unnötig zweimal eröffnet.

Th. weist in diesem Zusammenhang auch auf das oben erörterte gleichzeitige und unabhängige Vorkommen von Ohreneiterung hin: "it is to be remembered, that otitis may coineidently be present."

Ich bin am Scblusse, dieser heißt:

Grenzgebiete berïhren sich einerseits durch die gegenseitige Beeinflussung ihrer Zustände, andrerseits durch die häufige Gleichartigkeit oder täuschende Ählichkeit der Symptome ihrer Erkrankungen. Nur genaueste Kenntnis jedes dieser Gebiete ist imstande, vorkommende Grenzüberschreitungen bald als solche festzustellen, so daf die vorliegende Erkrankung nach dem Orte ihrer Entstehung, nicht nach dem ihrer Erscheinung gew ürdigt und behandelt werden kann.

\section{Literatur:}

1. Journal de mêdicine, chirurgie, pharmacie etc. par Roux, Paris 1762 , T. 17. S. 455 .

2. Handbuch der Chirurgie, Bd. 1. S. 189.

3. Traité des plaies de tête et de l'encéphalite. Paris 1835. S. 488. (Diese drei Zitate sind dem Handbuch der Chirurgie von V. Bruns, 1. Abteilung; Tübingen 1854 ; S. 976-978 entnommen.)

4. Brit. med. Journal, 1887 , I. S. 725 .

à. Bulletin de la société de chimrgie. 1873. 8. 507 .

6. Arehiv für Laryngologie, 1901.

7. Volkmanns klinische Vorträge. Nene Folge. Heft 1.

8. Handbuch der topographischen Anatomie. S. 300 .

9. Berliner klinische Wochenschrift, 1907.

10. Macew en, die infektiös-eitrigen Erkrankungen des Gehirns ete. S.112.

11. Z eller, zit. bei Oppenheim (24) S. 189.

12. Zeitschrift für Ohrenheilkunde, 1904. \$. 227.

13. Revue hebdomad. de laxyngol. ete. 1905. 15. Ref. Zentralbl. für Chir. 1906. 36. S. 978 .

14. Arehiv für Ohrenheilknnde, 1905. Bd. 64. S. 92.

15. of. 9 , Fall $V$. 
16. Archiv für Ohrenheilkunde, Bd, 35. S. 279. Fall XII.

17. Monatsschr. für Ohrenheilkunde etc., 1905. \$. 357 .

18. Beiträge zur klin. Chirurgie. 1899. S. ว26. Bd. 25.

19. Berl. klin. Wochenschrift, 1901. S. 639.

20. ef. $16, \mathrm{~S} .89$.

21. ef. 9, Fall III.

22. cf. 17, Diskussion.

23. Lehre von den Naseneiterungen, 2. Aufl. \$. 257 und S. 110

24. Oppenheim, die Encephalitis und der HirnabszeB. S. 136.

25. Brit, med. Journal, 1906. II. S. 768 . 